\title{
EXPOSURE TO SELF-REPORTED OCCUPATIONAL NOISE AND DIABETES - A CROSS-SECTIONAL RELATIONSHIP IN 7TH EUROPEAN SOCIAL SURVEY (ESS7, 2014)
}

\author{
ANGEL MARIO DZHAMBOV \\ Medical University of Plovdiv, Plovdiv, Bulgaria \\ Faculty of Public Health, Department of Hygiene and Ecomedicine
}

\begin{abstract}
Objectives: Almost nothing is known about the effect of occupational noise on diabetes, and this is particularly relevant given the wide spread of both noise exposure and diabetes. This study has aimed to determine whether occupational noise exposure is associated with higher risk of diabetes in Europe. Material and Methods: This study is based on 7th European Social Survey (ESS7, 2014) - a multi-country population-based questionnaire survey, which covered 28221 Europeans aged $\geq 15$ years old. Data on self-reported noise exposure, diabetes and other sociodemographic and work-related factors was available. The odds of prevalent diabetes were explored using unconditional logistic regression. Results: In the total sample $(\mathrm{N}=23486)$, participants ever exposed to very loud noise had no substantive increase in the odds of diabetes (odds ratio $(\mathrm{OR})=1.01,95 \%$ confidence interval $(\mathrm{CI})$ : $0.78-1.32)$. There were subgroups with non-significantly increased odds: men $(\mathrm{OR}=1.12,95 \% \mathrm{CI}: 0.87-1.45)$, the elderly $(\mathrm{OR}=1.09,95 \% \mathrm{CI}$ : $0.91-1.31)$, ethnic minority members $(\mathrm{OR}=1.55,95 \% \mathrm{CI}: 0.91-2.62)$, those with secondary education $(\mathrm{OR}=1.05,95 \% \mathrm{CI}: 0.78-1.41)$ and those living in small cities/towns (OR = 1.07, 95\% CI: 0.89-1.29). Low-skilled white-collar workers had OR = 1.34 (95\% CI: 1.09-1.64). Among participants employed during the preceding 5 years the odds were OR $=1.24$ (95\% CI: $0.95-1.61)$. Conclusions: Self-reported occupational noise was not associated with increased odds of prevalent diabetes in the total sample. Sensitivity analyses revealed some subgroups with non-significantly higher odds. Our results suggest that further delve into the relationship between occupational noise and diabetes is feasible and warranted. Int J Occup Med Environ Health 2017;30(4):537-551
\end{abstract}

Key words:

Noise, Diabetes mellitus, Occupational Exposure, Gender differences, White-collar workers, Cross sectional

\section{INTRODUCTION}

Occupational noise exposure is still very common across Europe. According to 6th European Working Conditions Survey, 27.6\% of the European workers in 2015 were exposed to noise so loud that they were required to raise their voice to talk to other people at least a quarter of the time [1]. Aside from hearing impairment, noise in the workplace is a risk factor for cardiovascular disease [2,3] and adverse pregnancy outcomes [4]. However, there is a dearth of evidence from occupational studies regarding the risk of diabetes. Such evidence would be of particular relevance to public health given the wide spread and social significance of diabetes. Nearly 350 million people in the world are diabetic [5], and their number is

Received: January 1, 2016. Accepted: May 12, 2016.

Corresponding author: Angel Mario Dzhambov, Medical University of Plovdiv, Faculty of Public Health, Department of Hygiene and Ecomedicine, Vassil Aprilov Blvd., 15A, 4002, Plovdiv, Bulgaria (e-mail: angelleloti@gmail.com). 
projected to reach 380 million by 2025 [6]. Diabetes also costs societies considerable financial losses in terms of both direct and indirect expenses [7]. Given that behavioral prevention through promotion of healthy lifestyle clearly fails to manage this pandemic spread, environmental risk factors should be targeted as well, but in order to do so, they should first be understood.

Heretofore only one case-control study specifically aimed to explore the risk of diabetes in relation to occupational noise exposure and found odds ratio $(\mathrm{OR})=1.04$ (95\% confidence interval (CI): 0.67-1.59) in the highest exposure group ( $>95 \mathrm{dBA}$-year), in reference to the lowest $(<85$ dBA-year) [8]. A meta-analysis, including that and other primary studies (which did not specifically focus on diabetes) concluded that there was no risk for workers exposed to $>85 \mathrm{~dB}$ (RR $=0.91,95 \%$ CI: 0.78-1.06) [9]. Conversely, according to some environmental health researchers, traffic noise is associated with an increased risk of incident diabetes [10,11] and obesity [12], although others have been inconclusive and failed to observe statistically significant effects [13].

There is a strong biological foundation for the purported relationship between noise and diabetes. The underlying pathophysiological mechanisms constitute the result of the stress response to ambient noise, which could lead to the increase in catecholamine and cortisol levels, elevated blood glucose, impaired glucose uptake and insulin sensitivity in the peripheral tissues $[9,10]$. Noise may also disturb normal sleep and adversely alter its phases; disturbance of sleep architecture modulates the central appetite control through the hunger hormones leptin and ghrelin, which could lead to obesity and impairment of carbohydrate metabolism $[12,14,15]$. Dzhambov has suggested that while sleep disturbance could explain the effect in residential environment, this hypothesis may not be applicable to occupational noise [9].

The evidence we currently have does not allow for making any inferences about the effect of occupational noise.
Moreover, Song's study had some validity issues with the adopted noise metric, its sample was smallish (202 cases and 1010 controls) and covered only Canada [8]. In order to advance the knowledge in this emerging field of occupational hygiene, we have aimed to determine whether occupational noise exposure is associated with higher risk of diabetes in Europe.

\section{MATERIAL AND METHODS}

\section{Study sample}

The source population for this study has been 7th round of the European Social Survey (ESS7). The ESS is a multicountry biennial repeated cross-sectional survey, aiming to "monitor and interpret changing public attitudes and values within Europe and to investigate how they interact with Europe's changing institutions." It employs strict random probability sampling of the population aged $\geq 15$ years old and resident within private households. The ESS7 was conducted in 2014 and covered 22 European countries, but in the currently available first edition (published October 28, 2015) data for 15 countries (Austria, Belgium, the Czech Republic, Denmark, Estonia, Finland, France, Germany, Ireland, Netherlands, Norway, Poland, Slovenia, Sweden and Switzerland) was released. The minimum target response rate was $70 \%$, and the actual, around $50-60 \%$, depending on the country. Self-reported data was collected during face-to-face interviews at participants' home. What makes the ESS7 unique and of particular interest is that it has introduced a panel on self-reported diagnoses with key non-communicable diseases as well as an occupational exposure panel [16].

\section{Ethics}

Each national organization involved in data collection for the ESS7 obtained participants' informed consent, adhered to the Declaration on Ethics of the International Statistical Institute and received ethics approval by local authorities [17]. The primary data was available 
for scientific research from the ESS website [18]. They were handled anonymously and thereby this secondary study was exempt from the institutional review board's approval [17].

\section{Assessment of health outcomes}

Self-reported diagnoses with diabetes and hypertension were ascertained by means of the question: "Which of the health problems on this card have you had or experienced in the last 12 months?" with two of the response options being "Diabetes" and "High blood pressure."

\section{Noise exposure assessment}

Participants were considered to be exposed to noise if they answered affirmatively to the question "And in any of the jobs you have ever had, which of the things on this card were you exposed to?" with one of the response options being "Very loud noise." The phrasing of the question was not very specific as it did not contain information on duration of exposure or whether it preceded the onset of diabetes. Therefore, before exploring the risk of diabetes, we tested the criterion validity of this question, according to the method proposed by Song [8]. The latter assumes that, since we know, based on many years of research, that objectively measured occupational noise is associated with an increased risk of hypertension, a valid self-reported noise question should be as well. Thus, in order for the results for diabetes to have credibility, participants who have worked under very loud noise should be at an increased risk of prevalent hypertension.

\section{Other covariates}

We additionally extracted information on other important factors. The sociodemographic panel included age, gender, ethnic minority status (yes vs. no), highest educational attainment and country of birth (born in the country currently living in or not). Educational attainment was initially coded according to the International
Standard Classification of Education (ISCED) [16] and re-coded into four categories for the analyses - lower secondary or less (ISCED I and II), upper secondary (ISCED IIIa and IIIb), advanced vocational/sub-degree (ISCED IV) and tertiary (ISCED V1 and V2). If it were not possible to harmonize the degree according to the ISCED, the cases were dropped. We also included the type of settlement (big city/suburbs, town/small city or countryside/village), since it might have reflected differences in neighborhood environment or job availability. Participants' household net income was categorized into deciles for each country and further collapsed into three categories for the analyses $-\leq 30$ percentile, $31-70$ percentile and $>70$ percentile.

Based on participants' job tile and description of their duties they were classified according to the International Standard Classification of Occupations 08 (ISCO-08) [16]. For the analyses, we collapsed the ISCO-08 codes into four categories - high skilled white-collar (ISCO codes 1, 2 and 3), low skilled white-collar (ISCO codes 4 and 5), high skilled blue-collar (ISCO codes 6 and 7) and low skilled bluecollar (ISCO codes 8 and 9) workers. Armed forces were excluded. The type of occupation was adjusted for as it represents other exposures in addition to noise, as well as the type of noise source (e.g., machine noise vs. noise from human voice). We also used information on whether participants had been in paid work and whether they had had any period of unemployment or work seeking during the last 5 years.

Another panel included individual and behavioral risk factors for diabetes. Body mass index was calculated from self-reported weight divided by height squared. Participants were allocated in one of the four groups, according to the World Health Organization (WHO) criteria [19]: < $18.50 \mathrm{~kg} / \mathrm{m}^{2}$ (underweight), 18.50-24.99 kg/m² (normal weight), $25-29.99 \mathrm{~kg} / \mathrm{m}^{2}$ (preobese) and $\geq 30 \mathrm{~kg} / \mathrm{m}^{2}$ (obese). The consumption of fruit and vegetables was coded as "every day," "1-6 times per week" and " $<1$ time 
per week." Cigarette-smoking status was categorized as "current-smoker" (currently smoking daily or not every day), "former-smoker" (does not smoke but used to or smoked only a few times) and "never-smoker." Alcoholdrinking status was categorized as " $\geq 1$ time per week," "1-3 times per month" and " $<1$ time per month." The number of days during the last week in which participants practiced sport/physical activity for $\geq 30 \mathrm{~min}$ was also extracted.

\section{Data analysis}

Data was first screened for missing values. For each of the analyses, cases with missing values were excluded. The univariate associations between the variables were examined with Pearson's Chi test and Student's t-test.

The associations between occupational noise and diabetes/hypertension were assessed by estimating prevalent odds ratios via unconditional logistic regression. Several models with increasing adjustments are reported for each outcome: basic model - adjusted for age and gender; main model - additionally adjusted for ethnicity, education, country of birth, type of settlement and occupation; and fully adjusted model - additionally adjusted for income, paid work, fruit consumption, vegetables consumption, smoking status, alcohol-drinking status, body mass index, sport/physical activity (and for diabetes when the outcome was hypertension). The main model adjusts for covariates with long-term validity whereas the fully adjusted model includes some temporally unstable factors. Therefore, due to the cross-sectional design of the ESS7, the key interpretation of the logistic regression is based on the main model, rather than the fully adjusted model.

In the sensitivity analyses, we run the models in subsamples stratified by gender, age group, educational attainment, ethnic minority status, type of settlement and occupational category. Interaction terms were tested formally at a relaxed $\alpha$ level of 0.25 in order not to miss any true effect modification.
It is recommended that the ESS7 data be weighted when running any analysis. Two types of weights are provided - design weights (correcting for unequal probabilities for selection) and population weights (correcting for country population size) [16]. Researchers have handled the weighting differently [20,21]; we used the product of the two weights (dweight $\times$ pweight) as sampling weight. Owing to the clustered structure of the data, standard errors clustered at the country level were used [22,23]. Results were considered statistically significant at $\mathrm{p}<0.05$ and odds ratios precise if the if the upper-to-lower $95 \%$ confidence limits ratio (CLR) was $<2.00[24]$.

\section{RESULTS}

The dataset contains a total of 28221 cases. Two thousand and fifty-one participants were missing information on diabetes and hypertension, 2436 - on occupational category and 5771 - on their income. Other missing rates were negligible and all participants had noise exposure data.

Sample characteristics are presented in the Table 1. Women were slightly more; different age groups were represented equally. Most participants were born in the respective country and did not pertain to an ethnic minority. Nearly $40 \%$ had completed upper secondary education. Most participants lived in smaller settlements. Almost one-third $(27.25 \%)$ reported having ever worked in a job under very loud noise, which is consistent with the results from 6th European Working Conditions Survey (27.6\%) [1].

The Table 1 also shows the univariate associations between different factors and participants diabetes status. About 5\% reported diagnosis with diabetes. Diabetics were older, with lower educational degree, lower income and with hypertension. They also consumed fruit more often, drank alcohol less often, exercised less often and were more likely to be overweight or obese. Fewer diabetics were current-smokers, but significantly more were former-smokers. With respect to occupation, they were 
Table 1. Sample characteristics and their univariate associations with respondents' diabetes status

\begin{tabular}{|c|c|c|c|c|}
\hline \multirow[b]{2}{*}{ Characteristics } & \multicolumn{3}{|c|}{ Respondents } & \multirow[b]{2}{*}{$\mathrm{p}$} \\
\hline & $\begin{array}{c}\text { total } \\
(\mathrm{N}=28221)^{\mathrm{b}}\end{array}$ & $\begin{array}{l}\text { no diabetes } \\
(\mathrm{N}=24799)^{\mathrm{c}}\end{array}$ & $\begin{array}{c}\text { diabetes } \\
(\mathrm{N}=1371)^{\mathrm{c}}\end{array}$ & \\
\hline Respondents (total) [\%] & 100.00 & 94.89 & 5.12 & \\
\hline Men [\%] & 48.59 & 48.48 & 51.47 & 0.147 \\
\hline Age [\%] & & & & $<0.001$ \\
\hline$\leq 35$ years & 28.83 & 30.20 & 3.55 & \\
\hline $36-45$ years & 16.81 & 14.47 & 4.84 & \\
\hline $46-55$ years & 18.89 & 19.19 & 13.61 & \\
\hline $56-65$ years & 16.29 & 15.65 & 28.00 & \\
\hline$>65$ years & 19.18 & 17.49 & 50.00 & \\
\hline Ethnic minority members [\%] & 4.53 & 4.47 & 3.74 & 0.374 \\
\hline Born in the country [\%] & 90.42 & 90.45 & 90.68 & 0.855 \\
\hline Education [\%] & & & & $<0.001$ \\
\hline lower secondary or less & 24.62 & 24.06 & 35.78 & \\
\hline upper secondary & 39.59 & 39.55 & 40.75 & \\
\hline advanced vocational/sub-degree & 14.65 & 14.82 & 11.12 & \\
\hline tertiary (B.A., M.A., Ph.D.) & 21.14 & 21.58 & 12.35 & \\
\hline Income $[\%]$ & & & & $<0.001$ \\
\hline$\leq 30$ percentile & 25.63 & 24.89 & 38.66 & \\
\hline $31-70$ percentile & 43.29 & 43.35 & 42.20 & \\
\hline$>70$ percentile & 31.08 & 31.76 & 19.14 & \\
\hline Settlement [\%] & & & & 0.723 \\
\hline big city/suburbs & 28.02 & 27.96 & 27.55 & \\
\hline town/small city & 32.22 & 32.10 & 33.62 & \\
\hline countryside/village & 39.76 & 39.94 & 38.83 & \\
\hline Hypertension [\%] & 17.74 & 15.81 & 53.59 & $<0.001$ \\
\hline Fruit consumption [\%] & & & & 0.001 \\
\hline every day & 68.03 & 67.67 & 74.81 & \\
\hline 1-6 times/week & 24.54 & 24.89 & 17.80 & \\
\hline$<1$ time/week & 7.43 & 7.44 & 7.39 & \\
\hline Vegetables consumption [\%] & & & & 0.725 \\
\hline every day & 71.65 & 71.69 & 71.05 & \\
\hline 1-6 times/week & 25.68 & 25.63 & 26.62 & \\
\hline$<1$ time/week & 2.67 & 2.68 & 2.32 & \\
\hline Alcohol-drinking status [\%] & & & & $<0.001$ \\
\hline$\geq 1$ time/week & 44.56 & 45.01 & 37.92 & \\
\hline $1-3$ times/month & 23.28 & 23.58 & 17.05 & \\
\hline$<1$ time/month & 32.16 & 31.40 & 45.03 & \\
\hline
\end{tabular}


Table 1. Sample characteristics and their univariate associations with respondents' diabetes status - cont.

\begin{tabular}{|c|c|c|c|c|}
\hline \multirow[b]{2}{*}{ Characteristics } & \multicolumn{3}{|c|}{ Respondents } & \multirow[b]{2}{*}{$\mathrm{p}$} \\
\hline & $\begin{array}{c}\text { total } \\
(\mathrm{N}=28221)^{\mathrm{b}}\end{array}$ & $\begin{array}{c}\text { no diabetes } \\
\left(\mathrm{N}=24 \text { 799 }{ }^{\mathrm{c}}\right.\end{array}$ & $\begin{array}{c}\text { diabetes } \\
(\mathrm{N}=1371)^{\mathrm{c}}\end{array}$ & \\
\hline Smoking status [\%] & & & & $<0.001$ \\
\hline current-smoker & 25.61 & 25.93 & 19.74 & \\
\hline former-smoker & 34.14 & 33.77 & 41.34 & \\
\hline never-smoker & 40.25 & 40.30 & 38.92 & \\
\hline Sport/physical activity [days/week] $(\mathrm{M} \pm \mathrm{SE})$ & $3.13 \pm 0.02$ & $3.15 \pm 0.03$ & $2.54 \pm 0.10$ & $<0.001$ \\
\hline Body mass index $[\%]$ & & & & $<0.001$ \\
\hline$<18.50 \mathrm{~kg} / \mathrm{m}^{2}$ & 3.20 & 3.36 & 0.31 & \\
\hline $18.50-24.99 \mathrm{~kg} / \mathrm{m}^{2}$ & 47.87 & 49.39 & 19.87 & \\
\hline $25.00-29.99 \mathrm{~kg} / \mathrm{m}^{2}$ & 33.93 & 33.76 & 37.00 & \\
\hline$\geq 30.00 \mathrm{~kg} / \mathrm{m}^{2}$ & 15.00 & 13.50 & 42.82 & \\
\hline Occupation [\%] & & & & 0.002 \\
\hline high skilled white-collar & 42.93 & 43.27 & 36.47 & \\
\hline low skilled white-collar & 25.55 & 25.60 & 25.29 & \\
\hline high skilled blue-collar & 15.27 & 15.11 & 18.07 & \\
\hline low skilled blue-collar & 16.25 & 16.03 & 20.18 & \\
\hline Ever worked under very loud noise [\%] & 27.25 & 27.24 & 27.45 & 0.906 \\
\hline
\end{tabular}

Percentages are weighted and countries are treated as strata.

${ }^{a}$ The number of days during the last week on which participants practiced sport/physical activity for $\geq 30 \mathrm{~min}$.

${ }^{\mathrm{b}}$ Percentages may not add-up to $100 \%$ due to missing data.

c The univariate associations between diabetes and the other factors are tested in complete-case analyses.

$\mathrm{M}$ - mean; SE - standard error.

mostly blue-collar workers, and the association with noise was not significant.

The associations between noise exposure and other contextual factors are given in the Table 2. Noise-exposed participants were mostly male, middle-aged, with lower educational attainment (upper secondary and advanced vocational/sub-degree), living in the countryside/village and their job description classified them as blue-collar workers. Looking at the unweighted correlations with other occupational exposures, the highest was with vibration $\left(r_{s}=0.54\right)$, and the others were around 0.30.4 (data not shown). These results are congruent with what might be expected from objectively measured noise exposure.
However, to further examine the criterion validity of selfreported noise, we conducted logistic regression models with hypertension as a dependent variable (Table 3). In line with the accumulated scientific evidence about the cardiovascular effects of noise, self-reported exposure to loud occupational noise was associated with $27 \%$ (95\% CI: 10 $45 \%$ ) increased odds of hypertension. Interaction terms significant at $p<0.25$ suggested higher OR among lowskilled white collar workers, those with upper secondary education and not pertaining to an ethnic minority.

The Table 4 shows the final results from the logistic regressions with diabetes as a dependent variable. In the total sample, noise had no substantive effect on diabetes $(\mathrm{OR}=1.01,95 \%$ CI: 0.78-1.32, CLR = 1.69). Sensitivity 
Table 2. Sample characteristics and their univariate associations with respondents' exposure to very loud occupational noise

\begin{tabular}{|c|c|c|c|}
\hline \multirow{2}{*}{ Characteristics } & \multicolumn{2}{|c|}{$\begin{array}{c}\text { Respondents } \\
{[\%]}\end{array}$} & \multirow{2}{*}{$\mathrm{p}$} \\
\hline & $\begin{array}{r}\text { never exposed } \\
(\mathrm{N}=20858)^{\mathrm{c}}\end{array}$ & $\begin{array}{l}\text { ever exposed } \\
(\mathrm{N}=7363)^{\mathrm{c}}\end{array}$ & \\
\hline Respondents (total) & 72.75 & 27.25 & \\
\hline Men & 40.89 & 69.17 & $<0.001$ \\
\hline Age & & & $<0.001$ \\
\hline$\leq 35$ years & 29.56 & 26.87 & \\
\hline $36-45$ years & 16.31 & 18.17 & \\
\hline $46-55$ years & 18.09 & 21.02 & \\
\hline $56-65$ years & 15.83 & 17.52 & \\
\hline$>65$ years & 20.21 & 16.42 & \\
\hline Ethnic minority members & 4.63 & 4.27 & 0.426 \\
\hline Born in the country & 90.06 & 91.38 & 0.045 \\
\hline Education & & & $<0.001$ \\
\hline lower secondary or less & 25.37 & 22.60 & \\
\hline upper secondary & 37.61 & 44.85 & \\
\hline advanced vocational/sub-degree & 14.13 & 16.02 & \\
\hline tertiary & 22.88 & 16.53 & \\
\hline Income & & & 0.049 \\
\hline$\leq 30$ percentile & 25.51 & 25.93 & \\
\hline $31-70$ percentile & 42.67 & 44.82 & \\
\hline$>70$ percentile & 31.83 & 29.25 & \\
\hline Settlement & & & $<0.001$ \\
\hline big city/suburbs & 28.79 & 25.95 & \\
\hline town/small city & 32.83 & 30.60 & \\
\hline countryside/village & 38.38 & 43.45 & \\
\hline Occupation & & & $<0.001$ \\
\hline high skilled white-collar & 45.73 & 36.24 & \\
\hline low skilled white-collar & 30.14 & 14.62 & \\
\hline high skilled blue-collar & 10.20 & 27.34 & \\
\hline low skilled blue-collar & 13.93 & 21.79 & \\
\hline
\end{tabular}

Explanations as in Table 1.

analyses, however, revealed that there were elevated odds among some subgroups. Men had $12 \%$ (95\% CI: $-13-$ $45 \%$, CLR $=1.67)$ increased odds, while among women they were not increased. People aged $65+$ years old had 9\% (95\% CI: -9-31\%, CLR = 1.44) increased odds. In contrast to the results for hypertension, the effect for diabetes reached 55\% (95\% CI: $-9-162 \%$, CLR $=2.88$ ) among ethnic minority members. Low skilled white-collar 
Table 3. Associations between self-reported very loud occupational noise exposure and prevalence of hypertension

\begin{tabular}{|c|c|c|c|c|c|c|}
\hline \multirow[b]{2}{*}{ Sample stratification } & \multicolumn{2}{|c|}{ Basic model $^{\mathrm{a}}$} & \multicolumn{2}{|c|}{ Main model ${ }^{\mathrm{b}}$} & \multicolumn{2}{|c|}{ Fully adjusted model ${ }^{c}$} \\
\hline & OR $(95 \% \mathrm{CI})$ & $\begin{array}{l}\text { respondents } \\
{[\mathrm{n}]}\end{array}$ & OR $(95 \% \mathrm{CI})$ & $\begin{array}{l}\text { respondents } \\
{[\mathrm{n}]}\end{array}$ & OR $(95 \% \mathrm{CI})$ & $\begin{array}{c}\text { respondents } \\
{[\mathrm{n}]}\end{array}$ \\
\hline Respondents (total) & $1.31(1.14-1.52)$ & 26102 & $1.27(1.10-1.45)$ & 23486 & $1.18(0.98-1.41)$ & 20130 \\
\hline \multicolumn{7}{|l|}{ Gender } \\
\hline men & $1.27(1.02-1.59)$ & 12674 & $1.25(0.99-1.59)$ & 11486 & $1.18(0.87-1.60)$ & 10001 \\
\hline women & $1.38(1.28-1.48)$ & 13428 & $1.33(1.21-1.46)$ & 12000 & $1.23(1.09-1.38)$ & 10129 \\
\hline \multicolumn{7}{|l|}{ Age } \\
\hline$\leq 35$ years & $1.62(1.07-2.43)^{*}$ & 7208 & $1.33(0.85-2.10)^{*}$ & 5661 & $1.59(1.12-2.25)^{*}$ & 4703 \\
\hline $36-45$ years & $1.45(1.09-1.94)$ & 4168 & $1.44(1.05-1.96)$ & 3975 & $1.17(0.99-1.38)$ & 3355 \\
\hline $46-55$ years & $1.41(1.14-1.75)$ & 4594 & $1.30(0.97-1.75)$ & 4405 & $1.26(0.77-2.07)$ & 3834 \\
\hline $56-65$ years & $1.00(0.89-1.11)$ & 4493 & $1.05(0.93-1.20)$ & 4295 & $1.01(0.90-1.12)$ & 3771 \\
\hline$>65$ years & $1.36(1.20-1.55)$ & 5639 & $1.32(1.16-1.50)$ & 5150 & $1.23(1.04-1.45)$ & 4408 \\
\hline \multicolumn{7}{|l|}{ Ethnic minority members } \\
\hline no & $1.34(1.16-1.56)$ & 24783 & $1.29(1.13-1.48)$ & 22574 & $1.21(1.02-1.44)$ & 19338 \\
\hline yes & $0.71(0.48-1.03)$ & 1075 & $0.59(0.33-1.03)^{*}$ & 912 & $0.41(0.18-0.91)^{*}$ & 765 \\
\hline \multicolumn{7}{|l|}{ Education } \\
\hline lower secondary or less & $1.47(1.22-1.77)$ & 6504 & $1.37(1.19-1.58)$ & 5209 & $1.25(1.04-1.50)$ & 4281 \\
\hline upper secondary & $1.32(1.12-1.56)$ & 9860 & $1.35(1.12-1.62)^{*}$ & 9106 & $1.26(0.96-1.66)$ & 7674 \\
\hline advanced vocational/sub-degree & $1.07(0.89-1.28)$ & 3797 & $1.04(0.83-1.30)$ & 3600 & $0.97(0.81-1.15)$ & 3041 \\
\hline tertiary (B.A., M.A., Ph.D.) & $1.30(1.02-1.66)$ & 5805 & $1.28(1.01-1.61)$ & 5571 & $1.26(1.06-1.50)$ & 4926 \\
\hline \multicolumn{7}{|l|}{ Settlement } \\
\hline big city/suburbs & $1.24(1.08-1.43)$ & 7983 & $1.22(1.05-1.43)$ & 7264 & $1.17(0.99-1.39)$ & 6295 \\
\hline town/small city & $1.49(1.07-2.08)$ & 7962 & $1.45(1.04-2.03)$ & 7127 & $1.35(1.02-1.78)^{*}$ & 6093 \\
\hline countryside/village & $1.24(1.05-1.46)$ & 10083 & $1.18(0.99-1.42)$ & 9095 & $1.07(0.85-1.35)$ & 7623 \\
\hline \multicolumn{7}{|l|}{ Occupation } \\
\hline high skilled white-collar & $1.34(1.21-1.47)$ & 10307 & $1.27(1.16-1.40)^{*}$ & 10203 & $1.17(1.06-1.29)$ & 8862 \\
\hline low skilled white-collar & $1.47(1.17-1.85)$ & 6371 & $1.46(1.20-1.86)$ & 6268 & $1.21(1.02-1.44)$ & 5175 \\
\hline high skilled blue-collar & $1.15(1.00-1.32)$ & 3358 & $1.13(0.99-1.30)$ & 3299 & $1.08(0.83-1.40)$ & 2720 \\
\hline low skilled blue-collar & $1.22(0.88-1.69)$ & 3772 & $1.28(0.92-1.78)$ & 3716 & $1.28(0.82-2.01)$ & 3088 \\
\hline
\end{tabular}

Models are weighted and clustered standard errors are used.

${ }^{\text {a }}$ Adjusted for age and gender.

${ }^{\mathrm{b}}$ Basic model + additionally adjusted for ethnicity, education, country of birth, type of settlement and occupation.

${ }^{\mathrm{c}}$ Main model + additionally adjusted for income, paid work, fruit consumption, vegetables consumption, smoking status, alcohol-drinking status, body mass index, sport/physical activity, diabetes.

OR - odds ratio; CI - confidence interval.

* Interaction term significant at $\mathrm{p}<0.25$. 
Table 4. Associations between self-reported very loud occupational noise exposure and prevalence of diabetes

\begin{tabular}{|c|c|c|c|c|c|c|}
\hline \multirow[b]{2}{*}{ Sample stratification } & \multicolumn{2}{|c|}{ Basic model $^{\mathrm{a}}$} & \multicolumn{2}{|c|}{ Main model ${ }^{\mathrm{b}}$} & \multicolumn{2}{|c|}{ Fully adjusted model ${ }^{c}$} \\
\hline & OR $(95 \% \mathrm{CI})$ & $\begin{array}{l}\text { respondents } \\
{[\mathrm{n}]}\end{array}$ & OR $(95 \% \mathrm{CI})$ & $\begin{array}{l}\text { respondents } \\
{[\mathrm{n}]}\end{array}$ & OR $(95 \% \mathrm{CI})$ & $\begin{array}{c}\text { respondents } \\
{[\mathrm{n}]}\end{array}$ \\
\hline Respondents (total) & $1.06(0.88-1.28)$ & 26102 & $1.01(0.78-1.32)$ & 23486 & $0.88(0.66-1.17)$ & 20130 \\
\hline \multicolumn{7}{|l|}{ Gender } \\
\hline men & $1.17(0.97-1.42)$ & 12674 & $1.12(0.87-1.45)$ & 11486 & $0.94(0.71-1.25)$ & 10001 \\
\hline women & $0.87(0.62-1.23)^{*}$ & 13428 & $0.87(0.59-1.26)$ & 12000 & $0.78(0.54-1.13)$ & 10129 \\
\hline \multicolumn{7}{|l|}{ Age } \\
\hline$\leq 35$ years & $0.10(0.02-0.46)^{*}$ & 7208 & $0.10(0.02-0.49)^{*}$ & 5661 & $0.15(0.03-0.66)^{*}$ & 4377 \\
\hline $36-45$ years & $0.84(0.56-1.25)$ & 4168 & $0.98(0.59-1.65)$ & 3975 & $0.94(0.49-1.81)$ & 3355 \\
\hline $46-55$ years & $0.99(0.64-1.53)$ & 4594 & $0.97(0.67-1.39)$ & 4405 & $0.93(0.66-1.32)$ & 3794 \\
\hline $56-65$ years & $0.97(0.65-1.44)$ & 4493 & $1.00(0.58-1.74)$ & 4295 & $0.76(0.39-1.48)$ & 3723 \\
\hline$>65$ years & $1.26(1.12-1.41)$ & 5639 & $1.09(0.91-1.31)$ & 5150 & $0.99(0.86-1.13)$ & 4408 \\
\hline \multicolumn{7}{|l|}{ Ethnic minority members } \\
\hline no & $1.05(0.82-1.33)$ & 24783 & $1.00(0.73-1.36)$ & 22574 & $0.87(0.64-1.18)$ & 19338 \\
\hline yes & $1.28(0.53-3.13)$ & 1075 & $1.55(0.91-2.62)$ & 912 & $1.05(0.59-1.89)$ & 765 \\
\hline \multicolumn{7}{|l|}{ Education } \\
\hline lower secondary or less & $0.99(0.78-1.25)$ & 6504 & $0.94(0.76-1.16)$ & 5209 & $0.85(0.72-1.01)$ & 4281 \\
\hline upper secondary & $0.97(0.73-1.28)$ & 9860 & $1.05(0.78-1.41)$ & 9106 & $0.90(0.67-1.21)$ & 7674 \\
\hline advanced vocational/sub-degree & $1.00(0.45-2.22)$ & 3797 & $1.02(0.37-2.84)$ & 3600 & $0.78(0.31-1.96)$ & 3041 \\
\hline tertiary (B.A., M.A., Ph.D.) & $1.12(0.61-2.06)$ & 5805 & $1.11(0.61-2.03)$ & 5571 & $0.94(0.47-1.86)$ & 4926 \\
\hline \multicolumn{7}{|l|}{ Settlement } \\
\hline big city/suburbs & $1.09(0.88-1.34)$ & 7983 & $1.01(0.74-1.38)$ & 7264 & $1.01(0.68-1.49)^{*}$ & 6295 \\
\hline town/small city & $1.01(0.86-1.19)$ & 7962 & $1.07(0.89-1.29)$ & 7127 & $0.94(0.72-1.23)$ & 6093 \\
\hline countryside/village & $1.13(0.63-2.02)$ & 10083 & $1.05(0.61-1.79)$ & 9095 & $0.84(0.57-1.23)$ & 7623 \\
\hline \multicolumn{7}{|l|}{ Occupation } \\
\hline high skilled white-collar & $1.25(0.82-1.91)$ & 10307 & $1.17(0.74-1.85)^{*}$ & 10203 & $0.93(0.56-1.55)^{*}$ & 8862 \\
\hline low skilled white-collar & $1.37(1.13-1.66)$ & 6371 & $1.34(1.09-1.64)$ & 6268 & $1.27(1.00-1.60)$ & 5175 \\
\hline high skilled blue-collar & $0.76(0.61-0.93)$ & 3358 & $0.73(0.53-1.01)$ & 3299 & $0.58(0.40-0.86)$ & 2720 \\
\hline low skilled blue-collar & $0.91(0.54-1.54)$ & 3772 & $0.95(0.57-1.60)$ & 3716 & $0.88(0.52-1.48)$ & 3088 \\
\hline \multicolumn{7}{|l|}{ Employment in the last 5 years } \\
\hline employed all the time & & & $1.24(0.95-1.61)$ & & $1.12(0.84-1.50)$ & 3377 \\
\hline
\end{tabular}

Models are weighted and clustered standard errors are used.

${ }^{a}$ Adjusted for age and gender.

${ }^{\mathrm{b}}$ Basic model + additionally adjusted for ethnicity, education, country of birth, type of settlement and occupation.

${ }^{\mathrm{c}}$ Main model + additionally adjusted for income, paid work, fruit consumption, vegetables consumption, smoking status, alcohol-drinking status, body mass index, sport/physical activity.

Abbreviations as in Table 3. 
workers had the only statistically significant effect: $34 \%$ (95\% CI: 9-64\%, CLR = 1.50). Other subgroups with elevated odds of diabetes and narrow $95 \%$ CI were those with upper secondary education $(\mathrm{OR}=1.05 \%, 95 \% \mathrm{CI}$ : 0.78-1.41, CLR = 1.81) and those living in small cities/ towns $(\mathrm{OR}=1.07,95 \% \mathrm{CI}: 0.89-1.29, \mathrm{CLR}=1.45)$. Among participants employed during the preceding 5 years, the odds were $24 \%$ (95\% CI: $-5-61 \%$, CLR $=1.69$ ). The only materially significant effect in the fully adjusted model was among low skilled white-collar workers and those employed during the preceding 5 years.

\section{DISCUSSION}

This study investigated the effect of self-reported occupational noise exposure on the odds of prevalent diabetes in Europe. Overall, findings suggested that although the risk appeared to be negligible in the total sample, within some subgroups it was increased. Those included men, the elderly, ethnic minority members, low skilled white-collar workers, small settlement dwellers and those with lower education. While most point estimates were not statistically significant, their precision in terms of the CLR might be more important than statistical significance, according to some epidemiologists, as "[t]he estimates least influenced by chance are not those with low p-values, but those with narrow confidence intervals" [24]. According to Babisch, many noise studies report non-significant results and the "mechanistical application of the significance criterion is rejected" because even non-significant results with narrow 95\% CI have practical importance for public health [25]. Nevertheless, the dominance of statistical significance testing is still widespread, and the ORs we found were $<2$, indicating smallish effect, thereby, from conventional standpoint, our study does not support the hypothesis that self-reported occupational noise is associated with diabetes.

The overall effect in the only previous occupational study on noise and diabetes was OR $=1.04$ (95\% CI: 0.67-1.59) for those exposed to $>95 \mathrm{dBA}-y e a r$ (vs. $<85 \mathrm{dBA}$-year), but the point estimate was not very precise $(\mathrm{CLR}=2.37)$ [8]. Furthermore, the criterion validity of the employed noise metric in that study could be questioned, given that, counterintuitively, exposure to $>95 \mathrm{dBA}$-year was not associated with an increased risk of hypertension [8].

Regarding gender differences, Song used the matched case-control design and did not report separate results for men and women or other subgroups [8]. Overall, few occupational studies compared genders with respect to the cardiovascular effect of noise, and Gan et al. also pointed to a higher risk in men [26]. Looking at environmental research, Sørensen et al. found slightly higher risk of incident diabetes among women $\left(\operatorname{IRR}_{\text {per } 10 \mathrm{~dB}}=1.11,95 \% \mathrm{CI}: 1.03-1.20\right)$ in comparison to men $\left(\operatorname{IRR}_{\text {per } 10 \mathrm{~dB}}=1.05,95 \%\right.$ CI: 0.98-1.13) [10]. Clark et al. followed a cohort of 380738 participants and estimated $18 \%$ higher likelihood of incident diabetes per $20 \mathrm{~dB}$ increase of road traffic noise [11]. Research on obesity and traffic noise yielded controversial results - Pyko et al. reported higher risk in men [14] whereas Christensen et al. did not report significant differences [15]. We hypothesize that, while in residential settings gender-specific effects may be attributed to differences in stress response [27] and sleep architecture [28], in occupational epidemiology they are also due to the different type of exposure in terms of noise source, frequency, duration, and other coexposures. Both diabetics and noise-exposed individuals are more often male.

As regards the other sensitivity results, in line with our findings, Sørensen et al. found higher risk of diabetes in participants aged $65+$ years old and those with lower education [10]. Further comparisons with occupational studies were not possible because of the general lack of research on diabetes as well as because its focus on bluecollar and male workers $[2,3]$.

Ethnic minorities have previously been given little consideration. Sbihi et al., for example, found that Sikhs and 
Chinese sawmill workers had substantively higher risk of noise-attributed hypertension than those from the white race [29]. Since participants' noise exposure did not differ across ethnic groups, differences in the effect size could be attributed to some unaccounted for residual confounding such as nutritional habits, religious practices, access to healthcare [30], environmental sensitivity or sleep quality [31,32], which could be explored by additional analyses of the dataset. The literature also suggests ethnic and racial differences in the prevalence of hearing loss and hearing threshold levels $[33,34]$, which could moderate the effects of noise.

As for the highest risk being among low skilled whitecollar workers (clerks and service workers), they might be exposed to high levels of social stress in addition to noise, and also not be able to wear hearing protectors due to the nature of their job. Stockholm et al. also concluded that among blue-collar workers the increase of noise exposure was not associated with an increased risk of hypertension [35].

In collusion, little is currently known about the effects of noise on the risk of diabetes, let alone in the occupational exposure literature.

\section{Strengths and limitations}

This was the second study in the field of occupational noise and diabetes and the first one to cover a representative multi-country population. Moreover, the sample was large ( $>20000$ ), in comparison to that used by Song (202 cases and 1010 controls) [8], and drawn from the general population, which is uncommon in occupational noise studies, typically limited to selected industries and occupations [36].

Nevertheless, there are some important limitations. First, the cross-sectional design of the ESS7 and the non-specific noise question mean that we could not determine the temporal relationship between exposure and outcome and for how many years were participants exposed. With respect to the cardiovascular effects of noise, it has been argued that it is unlikely that people with the outcome (in our case, diabetes) will purposefully get exposed (into a noisy job), while there is sufficient biological evidence for the opposite [37]. By stratifying the analyses by age, we assumed that the latter was a proxy for the length of service, which was unknown. Limiting the analyses to participants employed during the past 5 years was associated with increased odds of diabetes. Moreover, our interpretation of the results is based on the main model adjusted for covariates with long-term validity because the diet and physical activity might have been modified as a therapeutic strategy to manage diabetes. Still, prospective studies are needed to elucidate this relationship.

The second issue is the validity of self-reported diabetes and exposure. For diabetes, several studies compared self-reported diagnosis with biomedical data and found moderate sensitivity $(\approx 60 \%)$ and high specificity $(>95 \%)$ depending on the question [38-40]. On the other hand, "very loud noise" implies a significant vocal effort to talk to others and is typically assumed to be a proxy for about $80-85 \mathrm{~dB}$ [41-43]. At these levels the risk of diabetes is biologically feasible [9]. Moreover, we have shown criterion validity of the measure by testing the risk of prevalent hypertension. Nevertheless, our results may only be interpreted qualitatively (i.e., exposed vs. never exposed) and could serve to generate a basis for future research into the metabolic effects of noise, rather than to establish a precise exposure-response relationship between the two. The exposure misclassification is likely to bias the results towards the null and makes them conservative.

Another issue, pertinent to most occupational epidemiology studies, is the healthy worker/survivor effect [44] workers coping ineffectively with noise stress might have changed their job or even experienced a fatal event before the onset of diabetes, while diabetes itself could have 
forced others to leave a noisy job; both scenarios might have additionally flattened the association between noise and diabetes.

The lack of information on residential exposures (e.g., traffic pollution) is rarely addressed in occupational epidemiology studies, as, strictly speaking, residential exposures do not meet the criteria for confounders. We attempted to account for neighborhood context by stratifying the analyses by the type of settlement. Finally, the fact that no effect was observed among blue-collar workers implies that the lack of information on the use of hearing protection in those occupations could have masked the true effect.

In summary, we contend that the strengths of this study and its relevance for occupational health research outweigh its limitations. Nevertheless, future research should attempt to correct for these limitations and focus on the allegedly vulnerable subgroups we suggested.

\section{CONCLUSIONS}

Occupational noise exposure was not associated with increased odds of diabetes in the total sample. Sensitivity analyses, however, revealed some subgroups with nonsignificantly higher odds of diabetes - men, the elderly, ethnic minority members, those with upper secondary education and those living in smaller settlements. Among low skilled white-collar workers the risk estimate had narrow confidence interval and was statistically significant. Our results suggest that further delve into the relationship between occupational noise and diabetes is feasible and warranted.

\section{ACKNOWLEDGMENTS}

I am grateful to the European Social Survey research team for making the data from the ESS7 available. However, I retain sole responsibility for the analyses, interpretations and conclusions based on this data, which do not necessarily represent the views of the European Social Survey research team.

\section{REFERENCES}

1. Eurofound [Internet]. Dublin: Eurofund; 2017 [cited 2015 Dec 22]. Sixth European Working Conditions Survey: 2015. Available from: https://www.eurofound.europa.eu/surveys/ european-working-conditions-surveys/sixth-european-working-conditions-survey-2015.

2. Van Kempen EE, Kruize H, Boshuizen HC, Ameling CB, Staatsen BA, de Hollander AE. The association between noise exposure and blood pressure and ischemic heart disease: A meta-analysis. Environ Health Perspect. 2002;110(3): 307-17, https://doi.org/10.1289/ehp.02110307.

3. Tomei G, Fioravanti M, Cerratti D, Sancini A, Tomao E, Rosati MV, et al. Occupational exposure to noise and the cardiovascular system: A meta-analysis. Sci Total Environ. 2010;408(4):681-9, https://doi.org/10.1016/j.scitotenv.20 09.10.071.

4. Dzhambov AM, Dimitrova DD, Dimitrakova ED. Noise exposure during pregnancy, birth outcomes and fetal development: Meta-analyses using quality effects model. Folia Med (Plovdiv). 2014;56(3):204-14, https://doi.org/10.2478/folmed2014-0030.

5. Danaei G, Finucane MM, Lu Y, Singh GM, Cowan MJ, Paciorek CJ, et al. National, regional, and global trends in fasting plasma glucose and diabetes prevalence since 1980: Systematic analysis of health examination surveys and epidemiological studies with 370 country-years and 2.7 million participants. Lancet. 2011;378:31-40, https://doi.org/10.1016/ S0140-6736(11)60679-X.

6. Van Dieren S, Beulens JW, van der Schouw YT, Grobbee DE, Neal B. The global burden of diabetes and its complications: An emerging pandemic. Eur J Cardiovasc Prev Rehabil. 2010;17 Suppl 1:S3-8, https://doi.org/10.1097/01. hjr.0000368191.86614.5a.

7. American Diabetes Association. Economic costs of diabetes in the U.S. in 2012. Diabetes Care. 2013;36:1033-46, https:// doi.org/10.2337/dc12-2625.

8. Song C. Occupational noise exposure and the risk of diabetes, rheumatoid arthritis, and cardiovascular disease [disserta- 
tion]. [Internet]. Vancouver: University of British Columbia; 2013 [cited 2016 Jan 12]. Available from: https://open.library. ubc.ca/cIRcle/collections/ubctheses/24/items/1.0071919.

9. Dzhambov AM. Long-term noise exposure and the risk for type 2 diabetes: A meta-analysis. Noise Health. 2015; 17(74):23-33, https://doi.org/10.4103/1463-1741.149571.

10. Sørensen M, Andersen ZJ, Nordsborg RB, Becker T, Tjønneland A, Overvad K, et al. Long-term exposure to road traffic noise and incident diabetes: A cohort study. Environ Health Perspect. 2013;121(2):217-22, https://doi.org/ 10.1289/ehp.1205503.

11. Clark C, Tamburic L, Brauer M, Davies H. A prospective cohort study of road traffic noise effects on diabetes. In: Institute of Noise Control Engineering. INTER-NOISE and NOISE-CON Congress and Conference Proceedings; 2015 Aug 10; San Francisco, United States. Reston: The Institute; 2015. p. 5461-7.

12. Christensen JS, Raaschou-Nielsen O, Tjønneland A, Nordsborg RB, Jensen SS, Sørensen TI, et al. Long-term exposure to residential traffic noise and changes in body weight and waist circumference: A cohort study. Environ Res. 2015;143(Pt A):154-61, https://doi.org/10.1016/j.envres. 2015.10.007.

13. Eriksson C, Hilding A, Pyko A, Bluhm G, Pershagen G, Östenson CG. Long-term aircraft noise exposure and body mass index, waist circumference, and type 2 diabetes: A prospective study. Environ Health Perspect. 2014;122(7):68794, https://doi.org/10.1289/ehp.1307115.

14. Pyko A, Eriksson C, Oftedal B, Hilding A, Östenson CG, Krog NH, et al. Exposure to traffic noise and markers of obesity. Occup Environ Med. 2015;72(8):594-601, https:// doi.org/10.1136/oemed-2014-102516.

15. Christensen JS, Raaschou-Nielsen O, Tjønneland A, Overvad K, Nordsborg RB, Ketzel M, et al. Road traffic and railway noise exposures and adiposity in adults: A cross-sectional analysis of the Danish diet, cancer, and health cohort. Environ Health Perspect. 2016;124(3):329-35, https://doi. org/10.1289/ehp.1409052.
16. European Social Survey. ESS round 7: European Social Survey round 7 (2015): ESS-7 2014 documentation report. Edition 1.0. Bergen: European Social Survey Data Archive; 2015.

17. Van der Star A, Bränström R. Acceptance of sexual minorities, discrimination, social capital and health and well-being: A cross-European study among members of same-sex and opposite-sex couples. BMC Public Health. 2015;15(1):812, https://doi.org/10.1186/s12889-015-2148-9.

18. European Social Survey. ESS round 7: European Social Survey round 7 data (2014). Data file edition 1.0. Bergen: Norwegian Social Science Data Services; 2014.

19. World Health Organization. Obesity: Preventing and managing the global epidemic. Report of a WHO consultation. WHO technical report series No. 894. Geneva: The Organization; 2000.

20. Delaruelle K, Buffel V, Bracke P. Educational expansion and the education gradient in health: A hierarchical ageperiod-cohort analysis. Soc Sci Med. 2015;145:79-88, https:// doi.org/10.1016/j.socscimed.2015.09.040.

21. Cylus J, Papanicolas I. An analysis of perceived access to health care in Europe: How universal is universal coverage? Health Policy. 2015;119(9):1133-44, https://doi. org/10.1016/j.healthpol.2015.07.004.

22. Cameron AC, Miller DL. A practitioner's guide to clusterrobust inference. J Hum Res. 2015;50(2):317-73, https://doi. org/10.3368/jhr.50.2.317.

23. Primo DM, Jacobsmeier ML, Milyo J. Estimating the impact of state policies and institutions with mixed-level data. State Polit Policy Q. 2007;7(4):446-59, https://doi. org/10.1177/153244000700700405.

24. Poole C. Low P-values or narrow confidence intervals: Which are more durable? Epidemiology. 2001;12(3):291-4, https://doi.org/10.1097/00001648-200105000-00005.

25. Babisch W. Transportation noise and cardiovascular risk. Review and synthesis of epidemiological studies: Dose effect curve and risk estimation WaBoLu-Hefte [Internet]. 2006 [cited 2016 Jan 12];01/06. Available from: http://www.bruit. 
fr/images/stories/pdf/babisch_transportation_noise_cardiovascular_risk.pdf.

26. Gan WQ, Davies HW, Demers PA. Exposure to occupational noise and cardiovascular disease in the United States: The National Health and Nutrition Examination Survey 1999-2004. Occup Environ Med. 2011;68(3):183-90, https:// doi.org/10.1136/oem.2010.055269.

27. Verma R, Balhara YP, Gupta CS. Gender differences in stress response: Role of developmental and biological determinants. Ind Psychiatry J. 2011;20(1):4-10, https://doi. org/10.4103/0972-6748.98407.

28. Krishnan V, Collop NA. Gender differences in sleep disorders. Curr Opin Pulm Med. 2006;12(6):383-9, https://doi. org/10.1097/01.mcp.0000245705.69440.6a.

29. Sbihi H, Davies HW, Demers PA. Hypertension in noiseexposed sawmill workers: A cohort study. Occup Environ Med. 2008;65:643-6, https://doi.org/10.1136/oem.2007.035709.

30. Gee GC, Payne-Sturges DC. Environmental health disparities: A framework integrating psychosocial and environmental concepts. Environ Health Perspect. 2004;112:1645-53, https://doi.org/10.1289/ehp.7074.

31. Stepnowsky CJ Jr., Moore PJ, Dimsdale JE. Effect of ethnicity on sleep: Complexities for epidemiologic research. Sleep. 2003;26(3):329-32.

32. Kingsbury JH, Buxton OM, Emmons KM. Sleep and its relationship to racial and ethnic disparities in cardiovascular disease. Curr Cardiovasc Risk Rep. 2013;7(5):387-94, https:// doi.org/10.1007/s12170-013-0330-0.

33. Henselman LW, Henderson D, Shadoan J, Subramaniam M, Saunders S, Ohlin D. Effects of noise exposure, race, and years of service on hearing in U.S. Army soldiers. Ear Hear. 1995;16(4):382-91, https://doi.org/10.1097/00003446199508000-00005.

34. Agrawal Y, Platz EA, Niparko JK. Prevalence of hearing loss and differences by demographic characteristics among US adults: Data from the National Health and Nutrition Examination Survey, 1999-2004. Arch Intern Med. 2008;168: 1522-30, https://doi.org/10.1001/archinte.168.14.1522.
35. Stokholm ZA, Bonde JP, Christensen KL, Hansen AM, Kolstad HA. Occupational noise exposure and the risk of hypertension. Epidemiology. 2013;24:135-42, https://doi. org/10.1097/EDE.0b013e31826b7f76.

36. Choi YH, Hu H, Tak S, Mukherjee B, Park SK. Occupational noise exposure assessment using O*NET and its application to a study of hearing loss in the US general population. Occup Environ Med. 2012;69(3):176-83, https://doi. org/10.1136/oem.2011.064758.

37. Babisch W, Pershagen G, Selander J, Houthuijs D, Breugelmans $\mathrm{O}$, Cadum E, et al. Noise annoyance - A modifier of the association between noise level and cardiovascular health? Sci Total Environ. 2013;452-3:50-7, https:/doi. org/10.1016/j.scitotenv.2013.02.034.

38. Molenaar EA, van Ameijden EJ, Grobbee DE, Numans ME. Comparison of routine care self-reported and biometrical data on hypertension and diabetes: Results of the Utrecht Health Project. Eur J Public Health. 2007;17(2):199-205, https://doi.org/10.1093/eurpub/ckl113.

39. Yuan X, Liu T, Wu L, Zou ZY, Li C. Validity of self-reported diabetes among middle-aged and older Chinese adults: The China Health and Retirement Longitudinal Study. BMJ Open. 2015;5(4):e006633, https://doi.org/10.1136/bmjopen2014-006633.

40. Schneider AL, Pankow JS, Heiss G, Selvin E. Validity and reliability of self-reported diabetes in the atherosclerosis risk in communities study. Am J Epidemiol. 2012;176(8):738-43, https://doi.org/10.1093/aje/kws156.

41. McBride D. Guideline for diagnosing occupational noiseinduced hearing loss. Part 1: Noise effects and duration [Internet]. Accident Compensation Corporation; 2010 [cited 2016 Jan 12]. Available from: http://www.acc.co.nz/PRD_ EXT_CSMP/groups/external_communications/documents/ reference_tools/wpc091005.pdf.

42. Smith PA, Davis A, Ferguson M, Lutman ME. The prevalence and type of social noise exposure in young adults in England. Noise Health. 2000;6(2):41-56. 
43. Lazarus H. Prediction of verbal communication is noise A review: Part 1. App Acoust. 1986;19(6):439-64, https:// doi.org/10.1016/0003-682X(86)90039-3.
44. Arrighi HM, Hertz-Picciotto I. The evolving concept of the healthy worker survivor effect. Epidemiology. 1994;5(2): 189-96, https://doi.org/10.1097/00001648-199403000-00009.

This work is available in Open Access model and licensed under a Creative Commons Attribution-NonCommercial 3.0 Poland License - http://creativecommons.org/ licenses/by-nc/3.0/pl/deed.en. 